\title{
Osteoarthritis and osteoporosis: correlations between two clinical entities
}

\author{
I. Bertoldi, B. Frediani \\ Centro per l'Osteoporosi e la Diagnosi Strumentale OsteoArticolare, Sezione di Reumatologia, \\ Università di Siena, Italy
}

$\mathrm{O}$ steoarthritis (OA) and osteoporosis (OP) are two common muscoloskeletal diseases, especially in the elderly, which have a significant socio-economic impact. Both conditions are multi-factorial. OP is a systemic disease of the skeleton characterized by reduced mass and qualitative alterations of the bone, leading to increased risk of fracture; $\mathrm{OA}$ is an imbalance in tissue homeostasis, which leads to cartilage deterioration, sclerosis of subchondral bone and osteophytosis.

As early as the 1970s, the first studies were carried out in order to evaluate the association between the two diseases. The study by Foss et al demonstrated that bone mineral density (BMD) was higher in patients with prostheses at the hip for OA than in those with femur fractures (1). Ten years later, Dequeker proposed the existence of two groups of patients who differed anthropometrically: the osteoporotic patient, a longitype with low BMI and little muscle mass, and the osteoarthritic patient, a brachytype, overweight, with increased fat and muscle mass and fewer fractures (2).

Later, other studies confirmed an inverse correlation between $\mathrm{OP}$ and $\mathrm{OA}$. In the Chingford study, a small increase in BMD was observed in middle-aged women with early radiological OA of the hands, knees and lumbar spine (3). In the Rotterdam study, radiographic OA was found to be associated with higher BMD and increased rate of bone loss (4).

On the other hand, other studies suggest that $\mathrm{OA}$ is not correlated with increased BMD. A study by Frediani et al. showed that the presence of OA is not always cor- related with an increase in bone density: patients affected by severe OA of the hand and spine did not show significantly higher levels of stiffness (US evaluation at the calcaneous bone) than those with lower Kellgren index OA (5). Lingaerd et al observed that a significant proportion of patients with end-stage OA had OP but this diagnosis may be missed unless BMD measurements are performed at sites distant from the joints affected by OA (6).

These conflicting results may be ascribed to various confounding factors, such as different enrollment criteria and different BMD measurementites.

Another interesting aspect is the relationship between the progression of OA and BMD, but only few studies have been carried out in this regard.

According to the Framingham study, an elevated BMD of the femoral neck correlates with an increased incidence of $\mathrm{OA}$, but limits its progression (7). Other authors sustained the existence of two forms of presentation and development of OA: one defined as hypertrophic, characterized by a prevalence of osteophytosis, and the other hypotrophic characterized by an irregular reduction of joint and disc space. According to these authors, patients with the hypotrophic form of OA of the hip more frequently show vertebral fractures, and patients with fractures are less likely to develop osteophytosis (8).

Furthermore, the reduction of disc space corresponds to a greater risk of vertebral fractures regardless of BMD and other fracture risk factors (Ofely study) (9). These observations suggest that there are $\overline{\text { Corresponding author: }}$ \|laria Bertoldi Centro per l'Osteoporosi e la Diagnosi Strumentale OsteoArticolare Sezione di Reumatologia

Università di Siena, Italy

E-mail: bertoldilaria@libero.it 
factors, other than the BMD, that play an important role for the determination of the fracture risk.

The bone composition is definitely one of them. There are studies investigating the correlation between bone turnover and the presence of OA.

Some of them suggest that subjects with $\mathrm{OA}$ of the hands and knees present a reduced bone turnover, while others have shown an increased turnover (high urinary CTX-I and NTX-I levels) in patients suffering from hip and knee OA. Furthermore, these indices were higher in subjects who presented more advanced forms of OA and more rapid radiographic progression of the disease $(10,11)$.

These studies reappraise the original consideration of OA as a pathological process that involves only cartilage, as now it is confirmed that all of the structures of the joint are involved, particularly the subchondral bone. It is not contradictory to speak of OP and sclerosis in OA, given that in the early stages there is an elevated osteoclastic activity, and later a thickening of the compact bone, with reduced bone mineralization and diminished elasticity, which may explain why BMD values are elevated with no accompanying improvement in bone quality. The central role of the osteo-cartilaginous tidemark in determining whether the cartilage ossifies or becomes hyaline has been demonstrated (12). All of these pathogenetic mechanisms are perfectly compatible with both hypotheses regarding the pathogenesis of OA, but also with the hypothesis of the existence of two forms of OA: the hypertrophic, osteophytic type, characterized by elevated BMD, osteoformative tidemark response, slower disease progression, reduced turnover and lower incidence of fractures, and the hypotrophic type, characterized by lower $\mathrm{BMD}$, increased turnover, higher incidence of fractures and disease progression, with a reduction in cartilage thickness and no compensatory tidemark response.

Hence, the question: could anti-resorptive and anabolic drugs, usually used in OP play a role in the treatment of OA? Animal studies have indicated that calcitonin re- duces osteophytosis, cartilage lesions and the levels of cartilage degradation markers (13), while a study conducted in humans showed that $1 \mathrm{mg} /$ day per os of calcitonin improves the Lequesne index and reduces cartilage degradation markers (14).

The use of risedronate in animals induced a slowing of cartilage degradation (15), while in humans with knee OA, at a dose of $15 \mathrm{mg} /$ day, it improved disease symptoms and reduced bone and cartilage markers in patients with high CTX-II levels (16). The results of studies on alendronate in vitro are inconsistent: some indicate that alendronate reduces subchondral remodeling but accelerates cartilage degeneration, while others demonstrate a reduction in both cartilage markers and the formation of osteophytes (17).

In humans, doses of 5-10 mg/day of alendronate for three years have been shown to lower the osteophyte score by $30 \%$ and the dorsal-lumbar spinal column disc space reduction score by $50 \%$ (18).

Data on the relationship between estrogens and OA are contradictory, but their cartilage-protective aspect prevails in the case of long-term therapy (19).

In vitro and human studies on strontium ranelate demonstrated a chondro-protective role, in terms of reduction of cartilage and bone markers, and progression of the disease in the spinal column (20). Recently, a phase III trial indicated that strontium ranelate $2 \mathrm{~g} /$ day slowed the radiological progression of knee OA, reduced pain and improved patients' mobility (21).

The osteoprotegerin/receptor activator of nuclear factor kappa-B ligand (OPG/ RANK/RANKL) system is a well-known regulatory system for bone remodeling, and it is also produced and expressed in healthy and OA chondrocytes (22). Increased serum RANKL/OPG ratio is correlated with disease severity in patients with primary knee OA. In an animal OA model, intraperitoneal administration of OPG inhibited cartilage degradation in vivo, and this effect was attributed to the improvement in subchondral bone quality (23). In contrast, OPG had no effect on cartilage in vitro (24). 
Finally, there are data on the possible use of teriparatide to block cartilage degeneration. It is established that parathyroid hormone $(\mathrm{PTH})$ induces matrix synthesis and suppresses maturation of chondrocytes via the type $1 \mathrm{PTH}$ receptor (25) and also PTH receptor is up-regulated in articular chondrocytes during $\mathrm{OA}$ in humans and in a mouse model of injury-induced knee OA $(26,27)$.

A recent preclinical study showed that teriparatide inhibit aberrant chondrocyte maturation and associated articular cartilage degeneration; the chondro-protective and chondro-regenerative effects of teriparatide correlated with decreased levels of type $\times$ collagen, Runx 2 , matrix metalloproteinase-13 and the c-terminal portion of an aggrecan (28).

Diverse experimental and observational data strongly support the relevant role of subchondral bone in OA pathogenesis. There may be an effect of bone quality in the development and/or progression of OA, which has been understood from the study of the relationship between OP and OA. Both high and low bone mass phenotypes may influence the incidence and/or progression of OA through their indirect effect on subchondral bone.

Therefore, it is reasonable to assume that drugs affecting bone remodeling may hold a prominent role as DMOADs (disease modifying osteoarthritis drug). However, the beneficial effect of these agents in animal or in vitro models has not been yet confirmed in clinical studies. Pharmacodynamic properties of each agent, different doses used in animals, critical pharmacokinetic drug characteristics, and the stage of OA evolution may partially explain the different effects of bone-acting drugs in experimental models of OA when compared with studies in humans.

Further studies on the clinical relationships between OA and OP may allow to better understanding the relationship between bone and cartilage.

Studies on the role of certain OP drug treatments may help to better understand the role of bone turnover in various forms of OA.

\section{REFERENCES}

1. Foss MV, Byers PD. Bone density, osteoarthritis of the hip, and fracture of the upper end of the femur. Ann Rheum Dis. 1972; 31: 259-64.

2. Dequeker J, Goris P, Uytterhoeven R. Osteoporosis and osteoarthritis. Anthropometric distinctions. JAMA. 1983; 249: 1448-5.

3. Hart DJ, Mootoosamy I, Doyle DV, Spector TD. The relationship between osteoarthritis and osteoporosis in the general population: the Chingford Study. Ann Rheum Dis. 1994; 53: $158-62$.

4. Burger H, van Daele PL, Odding E, et al. Association of radiographically evident osteoarthritis with higher bone mineral density and increased bone loss with age. The Rotterdam Study. Arthritis Rheum. 1996; 39: 81-6.

5. Frediani B, Allegri A, Falsetti P, et al. Juxtaarticular osteoporosis in arthritis and arthrosis: fan beam X-ray densitometry. Arth Rheum 1999; 42: S355.

6. Lingard EA, Mitchell SY, Francis RM, et al. The prevalence of osteoporosis in patients with severe hip and knee osteoarthritis awaiting joint arthroplasty. Age Ageing. 2010; 39: 234-9.

7. Zhang Y, Hannan MT, Chaisson CE, et al. Bone mineral density and risk of incident and progressive radiographic knee osteoarthritis in women: the Framingham Study. J Rheumatol. 2000; 27: 1032-7.

8. Schnitzler CM, Mesquita JM, Wane L. Bone histomorphometry of the iliac crest, and spinal fracture prevalence in atrophic and hypertrophic osteoarthritis of the hip. Osteoporos Int. 1992; 2: 186-94.

9. Sornay-Rendu E, Allard C, Munoz F, et al. Disc space narrowing as a new risk factor for vertebral fracture: the OFELY study. Arthritis Rheum. 2006; 54: 1262-9.

10. Garnero P, Aronstein WS, Cohen SB, et al. Relationships between biochemical markers of bone and cartilage degradation with radiological progression in patients with knee osteoarthritis receiving risedronate: the knee osteoarthritis structural arthritis randomized clinical trial. Osteoarthritis Cartilage. 2008; 16: 660-6.

11. Sowers M, Lachance L, Jamadar D, et al. The associations of bone mineral density and bone turnover markers with osteoarthritis of the hand and knee in pre- and perimenopausal women. Arthritis Rheum. 1999; 42: 483-9.

12. Muir P, McCarthy J, Radtke CL, et al. Role of endochondral ossification of articular cartilage and functional adaptation of the subchondral plate in the development of fatigue microcracking of joints. Bone. 2006; 38: 342-9.

13. Behets C, Williams JM, Chappard D, et al. Effects of calcitonin on subchondral trabecular bone changes and on osteoarthritic cartilage 
lesions after acute anterior cruciate ligament deficiency. J Bone Miner Res. 2004; 19: 1821-6.

14. Manicourt DH, Azria M, Mindeholm L, et al. Oral salmon calcitonin reduces Lequesne's algofunctional index scores and decreases urinary and serum levels of biomarkers of joint metabolism in knee osteoarthritis. Arthritis Rheum. 2006; 54: 3205-11.

15. Bingham CO 3rd, Buckland-Wright JC, Garnero $\mathrm{P}$, et al. Risedronate decreases biochemical markers of cartilage degradation but does not decrease symptoms or slow radiographic progression in patients with medial compartment osteoarthritis of the knee: results of the two-year multinational knee osteoarthritis structural arthritis study. Arthritis Rheum. 2006; 54: 3494-507.

16. Spector TD, Conaghan PG, Buckland-Wright JC, et al. Effect of risedronate on joint structure and symptoms of knee osteoarthritis: results of the BRISK randomized, controlled trial [ISRCTN01928173]. Arthritis Res Ther. 2005; 7: 625-33.

17. Ding M, Danielsen CC, Hvid I. The effects of bone remodeling inhibition by alendronate on three-dimensional microarchitecture of subchondral bone tissues in guinea pig primary osteoarthritis. Calcif Tissue Int. 2008; 82: 77-86.

18. Neogi T, Nevitt MC, Ensrud KE, et al. The effect of alendronate on progression of spinal osteophytes and disc-space narrowing. Ann Rheum Dis. 2008; 67: 1427-30.

19. De Klerk BM, Schiphof D, Groeneveld FP, et al. No clear association between female hormonal aspects and osteoarthritis of the hand, hip and knee: a systematic review. Rheumatology (Oxford). 2009; 48: 1160-5.

20. Alexandersen P, Karsdal MA, Byrjalsen I, Christiansen C. Strontium ranelate effect in postmenopausal women with different clinical levels of osteoarthritis. Climacteric. 2011; 14 : 236-43.

21. Cooper C, Reginster JY, Chapurlat R, et al. Efficacy and safety of oral strontium ranelate for the treatment of knee osteoarthritis: rationale and design of randomised, double-blind, placebo-controlled trial. Curr Med Res Opin. 2012; 28: 231-9.

22. Kwan Tat S, Amiable N, Pelletier JP, et al. Modulation of OPG, RANK and RANKL by human chondrocytes and their implication during osteoarthritis. Rheumatology (Oxford). 2009; 48: 1482-90.

23. Kadri A, Ea HK, Bazille C, et al. Osteoprotegerin inhibits cartilage degradation through an effect on trabecular bone in murine experimental osteoarthritis. Arthritis Rheum. 2008; 58: 2379-86.

24. Komuro H, Olee T, Kühn K, et al. The osteoprotegerin/receptor activator of nuclear factor kappaB/receptor activator of nuclear factor kappaB ligand system in cartilage. Arthritis Rheum. 2001; 44: 2768-76.

25. Kronenberg HM, Karaplis AC, Lanske B. Role of parathyroid hormone-related protein in skeletal development. Ann N Y Acad Sci. 1996; 785: 119-23.

26. Chen X, Macica CM, Nasiri A, Broadus AE. Regulation of articular chondrocyte proliferation and differentiation by indian hedgehog and parathyroid hormone-related protein in mice. Arthritis Rheum. 2008; 58: 3788-97.

27. Godler DE, Stein AN, Bakharevski O, et al. Parathyroid hormone-related peptide expression in rat collagen-induced arthritis. Rheumatology (Oxford). 2005; 44: 1122-31.

28. Sampson ER, Hilton MJ, Tian Y, et al. Teriparatide as a chondroregenerative therapy for injury-induced osteoarthritis. Sci Trans Med. 2011; 3: 101ra93. 